Statistics in the Twenty-First Century: Special Volume

In Honour of Distinguished Professor Dr. Mir Masoom Ali

On the Occasion of his 75th Birthday Anniversary

PJSOR, Vol. 8, No. 3, pages 479-489, July 2012

\title{
The Kumaraswamy-Inverse Weibull Distribution
}

\author{
Muhammad Qaiser Shahbaz \\ Department of Mathematics \\ COMSATS Institute of IT, Lahore, Pakistan \\ qshahbaz@gmail.com \\ Saman Shahbaz \\ Department of Mathematics \\ COMSATS Institute of IT, Lahore, Pakistan \\ samans@ciitlahore.edu.pk \\ Nadeem Shafique Butt \\ College of Statistical and Actuarial Sciences \\ University of the Punjab, Lahore, Pakistan \\ nadeemshafique@hotmail.com
}

\begin{abstract}
In this paper we have proposed a new four parameter Inverse Weibull distribution that is based upon the cumulative distribution function of Kumaraswamy (1980) distribution. The distributional properties of the proposed distribution have been studied. Special cases of the proposed distribution have also been explored.
\end{abstract}

Keywords: Kumaraswamy Distribution, Inverse Weibull Distribution, Generalized Distributions.

\section{Introduction}

The Beta distribution with density function:

$$
f_{B}(x)=\frac{1}{B(a, b)} x^{a-1}(1-x)^{b-1} ; \quad 0<x<1,(a, b) \in \mathfrak{R}^{+}
$$

is perhaps one of the most popular bounded continuous probability distribution. This distribution has attracted lot of attention within the area of theoretical and applied statistics. The distribution has found extensive application in areas including; but not limited to; engineering, computer sciences and hydrology. Eugene, Lee and Famoye (2002) have used the Beta distribution to generalize the Normal distribution. Eugene et al. (2002) has shown that the logit of Beta distribution can be used to generalize any parent cumulative distribution function $G(x)$ as:

$$
f_{B-G}(x)=I_{G(x)}(a, b)=\frac{1}{B(a, b)} \int_{0}^{G(x)} w^{a-1}(1-w)^{b-1} d w ;
$$

where $I_{x}(a, b)$ is incomplete Beta function ratio given as $I_{x}(a, b)=B_{x}(a, b) / B(a, b)$. 
Eugene et al. (2002) has used the CDF of Normal distribution in (1.2) to propose the Beta-Normal distribution. The generalization given in (1.2) has been used by number of authors to propose new distributions. Some notable references include Nadarajah and Kotz (2004, 2005), Famoye, Lee and Olumolade (2005), Hanook, Shahbaz, Mohsin and Kibria (2012) and many others.

Although the generalization of distribution functions given in (1.2) has attracted number of researchers, it still involves the complexity of incomplete beta function ratio. Some researchers have suggested to use other bounded distributions on $(0,1)$ to obtain the generalization of any parent cumulative distribution function. One such distribution is the Kumaraswamy (1980) distribution having density and distribution function as:

$$
f_{K}(x)=a b w^{a-1}\left(1-w^{a}\right)^{b-1} ; 0<x<1,(a, b) \in \mathfrak{R}^{+}
$$

and $\quad F_{K}(x)=1-\left(1-x^{a}\right)^{b}$.

Cordeiro and Castro (2010) have proposed a generalization of probability distributions by using (1.3) and (1.4). The density and distribution function of generalized class given by Cordeiro and Castro (2010) has following forms:

$$
f_{K-G}(x)=\operatorname{abg}(x)\{G(x)\}^{a-1}\left[1-\{G(x)\}^{a}\right]^{b-1} ;
$$

and $\quad F_{K-G}(x)=1-\left[1-\{G(x)\}^{a}\right]^{b}$.

Cordeiro, Ortega and Nadarajah (2010) have used the CDF of Weibull distribution in (1.5) to propose the Kumaraswamy-Weibull distribution. The generalization (1.5) and (1.6) can be used to propose other distributions based on the Kumaraswamy distribution.

In the following we have proposed the Kumaraswamy-Inverse Weibull (Kum-IW for short) distribution by using the CDF of Inverse Weibull distribution in (1.5).

\section{The Kumaraswamy-Inverse Weibull Distribution}

The Inverse Weibull distribution has been used as an effective model for failure data in the literature. The density and distribution function of Inverse Weibull random variable is given as:

$$
f_{I W}(x)=\frac{\alpha \beta}{x^{\beta+1}} \exp \left(-\frac{\alpha}{x^{\beta}}\right) ;(x, \alpha, \beta) \in \mathfrak{R}^{+} ;
$$

and $\quad F_{I W}(x)=\exp \left(-\frac{\alpha}{x^{\beta}}\right)$ 
The distribution (2.1) has been used by Hanook et al. (2012) to propose the Beta Inverse Weibull. We propose the Kumaraswamy-Inverse Weibull distribution by using the distribution function (2.2) in (1.5). The density function of Kum-IW distribution is given as:

$$
\begin{aligned}
f_{K-I W}(x) & =a b \frac{\alpha \beta}{x^{\beta+1}} \exp \left(-\frac{\alpha}{x^{\beta}}\right)\left\{\exp \left(-\frac{\alpha}{x^{\beta}}\right)\right\}^{a-1}\left[1-\left\{\exp \left(-\frac{\alpha}{x^{\beta}}\right)\right\}^{a}\right]^{b-1} \\
\text { or } \quad f_{K-I W}(x) & =\frac{a b \alpha \beta}{x^{\beta+1}} \exp \left(-\frac{a \alpha}{x^{\beta}}\right)\left[1-\exp \left(-\frac{a \alpha}{x^{\beta}}\right)\right]^{b-1} ;(x, a, b, \alpha, \beta) \in \mathfrak{R}^{+} .
\end{aligned}
$$

We will use the notation $\operatorname{KIW}(a, b, \alpha, \beta)$ to denote the density (2.3). Using following series representation of Prudnikov et al. (1986):

$$
(1+x)^{\alpha}=\sum_{j=0}^{\infty} \frac{\Gamma(\alpha+1)}{j ! \Gamma(\alpha+1-j)} x^{j}
$$

the density (2.3) can be written as:

$$
f_{K-I W}=\sum_{j=0}^{\infty}(-1)^{j} \frac{\Gamma(b)}{j ! \Gamma(b-j)} \cdot \frac{a b \alpha \beta}{x^{\beta+1}} \cdot \exp \left\{-\frac{a \alpha(j+1)}{x^{\beta}}\right\} .
$$

The density (2.4) can be viewed as an infinite weighted sum of Inverse Weibull densities. The distribution function of Kum-IW distribution is immediately written by using (1.6) as:

$$
F_{K-I W}(x)=1-\left[1-\exp \left(-\frac{a \alpha}{x^{\beta}}\right)\right]^{b}
$$

The graphs of density are shown below for various choices of the parameters. The plot shows that the parameter $\beta$ controls the kurtosis of the distribution. 
Figure 1: The plot of $K I W(a, b, \alpha, \beta)$ for various choices of the parameters

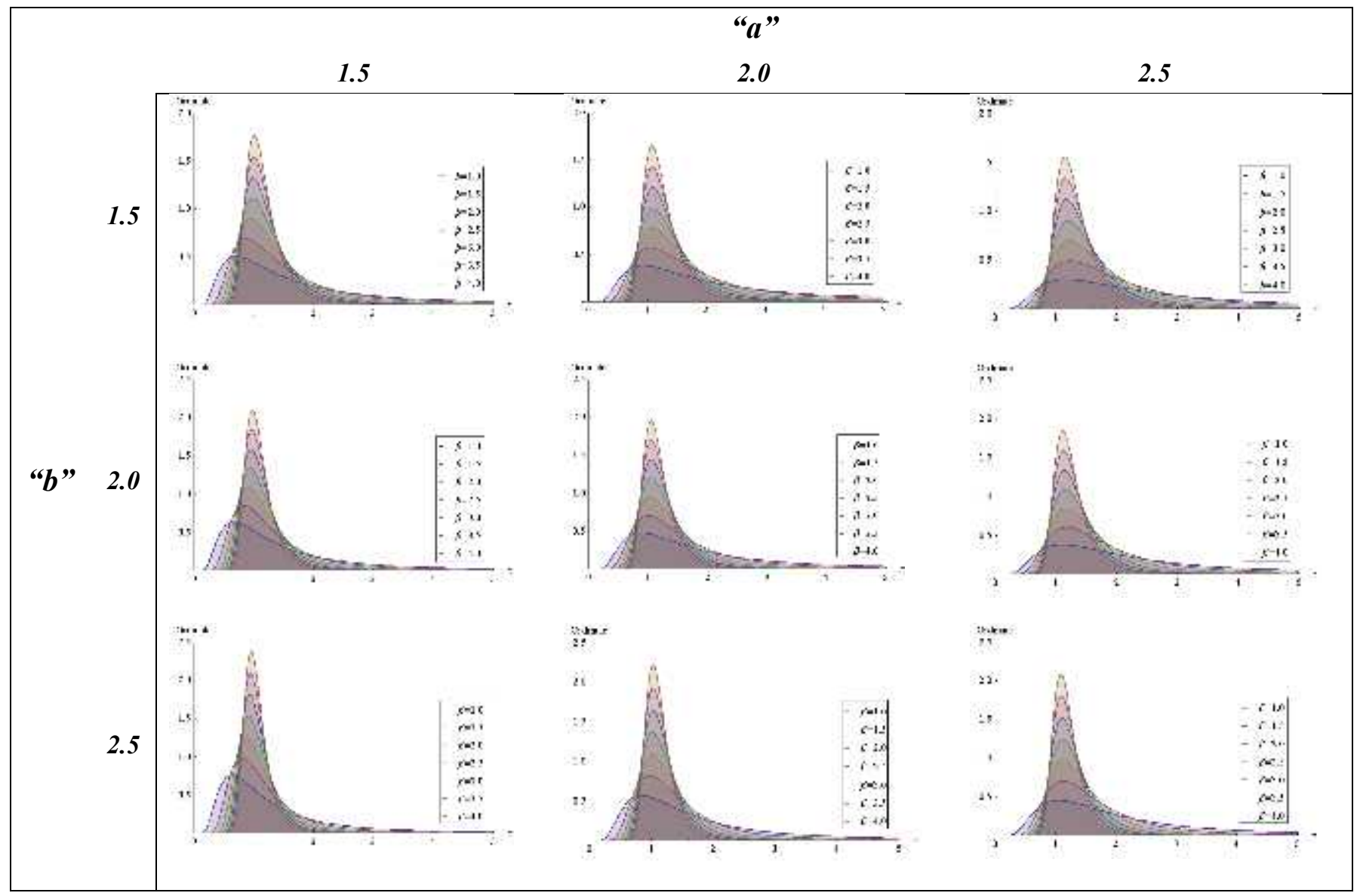

\section{Basic Properties of the Distribution}

In this section we have studied the basic properties of Kum-IW distribution. We first evaluate the moments of the proposed distribution as under:

$$
\begin{aligned}
\mu_{r}^{\prime}=E\left(X^{r}\right) & =\int_{0}^{\infty} x^{r} f_{K-I W}(x) d x \\
& =\int_{0}^{\infty} x^{r} \sum_{j=0}^{\infty}(-1)^{j} \frac{\Gamma(b)}{j ! \Gamma(b-j)} \cdot \frac{a b \alpha \beta}{x^{\beta+1}} \cdot \exp \left\{-\frac{a \alpha(j+1)}{x^{\beta}}\right\} d x \\
& =\sum_{j=0}^{\infty}(-1)^{j} \frac{a b \alpha \beta \Gamma(b)}{j ! \Gamma(b-j)} \int_{0}^{\infty} x^{r} \cdot \frac{1}{x^{\beta+1}} \cdot \exp \left\{-\frac{a \alpha(j+1)}{x^{\beta}}\right\} d x
\end{aligned}
$$

Making the transformation $w=a \alpha(j+1) / x^{\beta}$ in above expression and simplifying, the $r$-th moment of Kum-IW distribution is:

$$
\mu_{r}^{\prime}=\sum_{j=0}^{\infty}(-1)^{j} \frac{a b \alpha \beta \Gamma(b) \Gamma(1-r / \beta)}{j ! \beta[a \alpha(j+1)]^{1-r / \beta} \Gamma(b-j)} ;
$$

which exist for $r<\beta$. The mean, variance, Skewness and Kurtosis can be obtained from (3.1). Numerical values of Mean, Variance, Skewness and Kurtosis for various choices of parameters are given in Table 3.1 through Table 3.3. 
Table 3.1: Mean of Kum-IW distribution for various choices of parameters

\begin{tabular}{|c|c|c|c|c|c|c|}
\hline \multirow{2}{*}{$a$} & \multirow{2}{*}{$b$} & \multicolumn{5}{|c|}{$\beta$} \\
\hline & & 2 & 3 & 4 & 5 & 6 \\
\hline \multirow{5}{*}{1} & 1 & 1.7725 & 1.3541 & 1.2254 & 1.1642 & 1.1288 \\
\hline & 2 & 1.0383 & 1.0022 & 0.9936 & 0.9911 & 0.9906 \\
\hline & 3 & 0.8675 & 0.8971 & 0.9172 & 0.9310 & 0.9409 \\
\hline & 4 & 0.7851 & 0.8424 & 0.8760 & 0.8979 & 0.9133 \\
\hline & 5 & 0.7346 & 0.8074 & 0.8492 & 0.8761 & 0.8949 \\
\hline \multirow{5}{*}{2} & 1 & 2.5066 & 1.7061 & 1.4573 & 1.3374 & 1.2670 \\
\hline & 2 & 1.4684 & 1.2626 & 1.1816 & 1.1385 & 1.1119 \\
\hline & 3 & 1.2268 & 1.1303 & 1.0907 & 1.0694 & 1.0561 \\
\hline & 4 & 1.1102 & 1.0613 & 1.0417 & 1.0314 & 1.0251 \\
\hline & 5 & 1.0389 & 1.0173 & 1.0098 & 1.0064 & 1.0045 \\
\hline \multirow{5}{*}{3} & 1 & 3.0700 & 1.9530 & 1.6127 & 1.4503 & 1.3556 \\
\hline & 2 & 1.7984 & 1.4454 & 1.3076 & 1.2347 & 1.1896 \\
\hline & 3 & 1.5025 & 1.2938 & 1.2071 & 1.1597 & 1.1300 \\
\hline & 4 & 1.3598 & 1.2149 & 1.1529 & 1.1185 & 1.0968 \\
\hline & 5 & 1.2723 & 1.1645 & 1.1176 & 1.0914 & 1.0747 \\
\hline \multirow{5}{*}{4} & 1 & 3.5449 & 2.1495 & 1.7330 & 1.5362 & 1.4222 \\
\hline & 2 & 2.0766 & 1.5908 & 1.4051 & 1.3078 & 1.2480 \\
\hline & 3 & 1.7349 & 1.4240 & 1.2971 & 1.2284 & 1.1855 \\
\hline & 4 & 1.5701 & 1.3372 & 1.2388 & 1.1848 & 1.1506 \\
\hline & 5 & 1.4692 & 1.2817 & 1.2009 & 1.1560 & 1.1275 \\
\hline \multirow{5}{*}{5} & 1 & 3.9633 & 2.3155 & 1.8324 & 1.6063 & 1.4761 \\
\hline & 2 & 2.3217 & 1.7137 & 1.4857 & 1.3675 & 1.2953 \\
\hline & 3 & 1.9397 & 1.5340 & 1.3715 & 1.2845 & 1.2304 \\
\hline & 4 & 1.7554 & 1.4404 & 1.3099 & 1.2388 & 1.1942 \\
\hline & 5 & 1.6426 & 1.3806 & 1.2698 & 1.2088 & 1.1702 \\
\hline
\end{tabular}


Table 3.2: Variance of Kum-IW distribution for various choices of parameters

\begin{tabular}{|c|c|c|c|c|c|c|}
\hline \multirow{2}{*}{$a$} & \multirow{2}{*}{$b$} & \multicolumn{5}{|c|}{$\beta$} \\
\hline & & 3 & 4 & 5 & 6 & 7 \\
\hline \multirow{5}{*}{1} & 1 & 0.845 & 0.271 & 0.134 & 0.080 & 0.053 \\
\hline & 2 & 0.101 & 0.051 & 0.031 & 0.021 & 0.015 \\
\hline & 3 & 0.047 & 0.026 & 0.017 & 0.012 & 0.009 \\
\hline & 4 & 0.030 & 0.018 & 0.012 & 0.008 & 0.006 \\
\hline & 5 & 0.022 & 0.014 & 0.009 & 0.007 & 0.005 \\
\hline \multirow{5}{*}{2} & 1 & 1.342 & 0.383 & 0.176 & 0.101 & 0.065 \\
\hline & 2 & 0.160 & 0.072 & 0.041 & 0.026 & 0.018 \\
\hline & 3 & 0.074 & 0.037 & 0.022 & 0.015 & 0.011 \\
\hline & 4 & 0.048 & 0.025 & 0.015 & 0.010 & 0.008 \\
\hline & 5 & 0.035 & 0.019 & 0.012 & 0.008 & 0.006 \\
\hline \multirow{5}{*}{3} & 1 & 1.758 & 0.469 & 0.208 & 0.115 & 0.073 \\
\hline & 2 & 0.210 & 0.089 & 0.048 & 0.030 & 0.021 \\
\hline & 3 & 0.097 & 0.045 & 0.026 & 0.017 & 0.012 \\
\hline & 4 & 0.063 & 0.031 & 0.018 & 0.012 & 0.009 \\
\hline & 5 & 0.046 & 0.023 & 0.014 & 0.009 & 0.007 \\
\hline \multirow{5}{*}{4} & 1 & 2.130 & 0.542 & 0.233 & 0.127 & 0.079 \\
\hline & 2 & 0.255 & 0.102 & 0.054 & 0.033 & 0.022 \\
\hline & 3 & 0.118 & 0.053 & 0.029 & 0.019 & 0.013 \\
\hline & 4 & 0.076 & 0.035 & 0.020 & 0.013 & 0.009 \\
\hline & 5 & 0.056 & 0.027 & 0.016 & 0.010 & 0.007 \\
\hline \multirow{5}{*}{5} & 1 & 2.472 & 0.606 & 0.255 & 0.137 & 0.084 \\
\hline & 2 & 0.295 & 0.114 & 0.059 & 0.036 & 0.024 \\
\hline & 3 & 0.137 & 0.059 & 0.032 & 0.020 & 0.014 \\
\hline & 4 & 0.088 & 0.040 & 0.022 & 0.014 & 0.010 \\
\hline & 5 & 0.065 & 0.030 & 0.017 & 0.011 & 0.008 \\
\hline
\end{tabular}


Table 3.3: Skewness and Kurtosis of Kum-IW distribution for various choices of parameters

\begin{tabular}{|c|c|c|c|c|c|c|}
\hline \multirow{2}{*}{ a } & \multirow{2}{*}{$b$} & \multicolumn{5}{|c|}{$\beta$} \\
\hline & & 3 & 4 & 5 & 6 & 7 \\
\hline \multirow{5}{*}{ Skewness } & 1 & 5.605 & 3.535 & 2.806 & 2.425 & 2.189 \\
\hline & 2 & 1.776 & 1.502 & 1.342 & 1.237 & 1.163 \\
\hline & 3 & 1.194 & 1.037 & 0.940 & 0.873 & 0.825 \\
\hline & 4 & 0.927 & 0.809 & 0.734 & 0.682 & 0.643 \\
\hline & 5 & 0.766 & 0.667 & 0.603 & 0.558 & 0.525 \\
\hline \multirow{5}{*}{ Kurtosis } & 1 & 48.092 & 24.678 & 17.534 & 14.166 & 12.229 \\
\hline & 2 & 8.103 & 6.974 & 6.327 & 5.910 & 5.620 \\
\hline & 3 & 5.349 & 4.919 & 4.653 & 4.473 & 4.344 \\
\hline & 4 & 4.426 & 4.178 & 4.021 & 3.914 & 3.836 \\
\hline & 5 & 3.976 & 3.808 & 3.700 & 3.626 & 3.572 \\
\hline
\end{tabular}

Table 3.1 and 3.2 indicates that for fixed $a$ and $\beta$ the mean and variance of Kum-IW distribution will decrease with increase in $b$. Table 3.3 indicates that for fixed $a$ and $b$, the Skewness and Kurtosis both decreases with increase in $\beta$.

The $q$ th percentile of the distribution is obtained by solving $F(x)=q$ for the variable $X$. The $q$ th percentile for Kum-IW distribution is obtained by using (2.5) in $F(x)=q$, which turned out to be:

$$
x=\left[-\frac{a \alpha}{\ln \left\{1-(1-q)^{1 / b}\right\}}\right]^{1 / \beta} .
$$

The Median of the distribution is obtained by using $q=0.5$ in (3.2). The random sample can also be easily generated from (3.2) by using $q$ as uniform random number.

The survivorship function for Kum-IW distribution is immediately written from (2.5) and is given as:

$$
S_{K-I W}(t)=1-F_{K-I W}(t)=\left[1-\exp \left(-\frac{a \alpha}{x^{\beta}}\right)\right]^{b} .
$$


The hazard rate function of the distribution is:

$$
h_{K-I W}(t)=\frac{f_{K-I W}(t)}{1-F_{K-I W}(t)}=\frac{\frac{a b \alpha \beta}{x^{\beta+1}} \exp \left(-\frac{a \alpha}{x^{\beta}}\right)}{\left[1-\exp \left(-\frac{a \alpha}{x^{\beta}}\right)\right]} .
$$

The plots of (3.4), for different choices of the parameters, are given in figure 2 . These plots shows that for fixed $a$ and $b$, the hazard rate has higher peak for large values of $\beta$.

Figure 2: Hazard rate function of $\operatorname{KIW}(a, b, \alpha, \beta)$

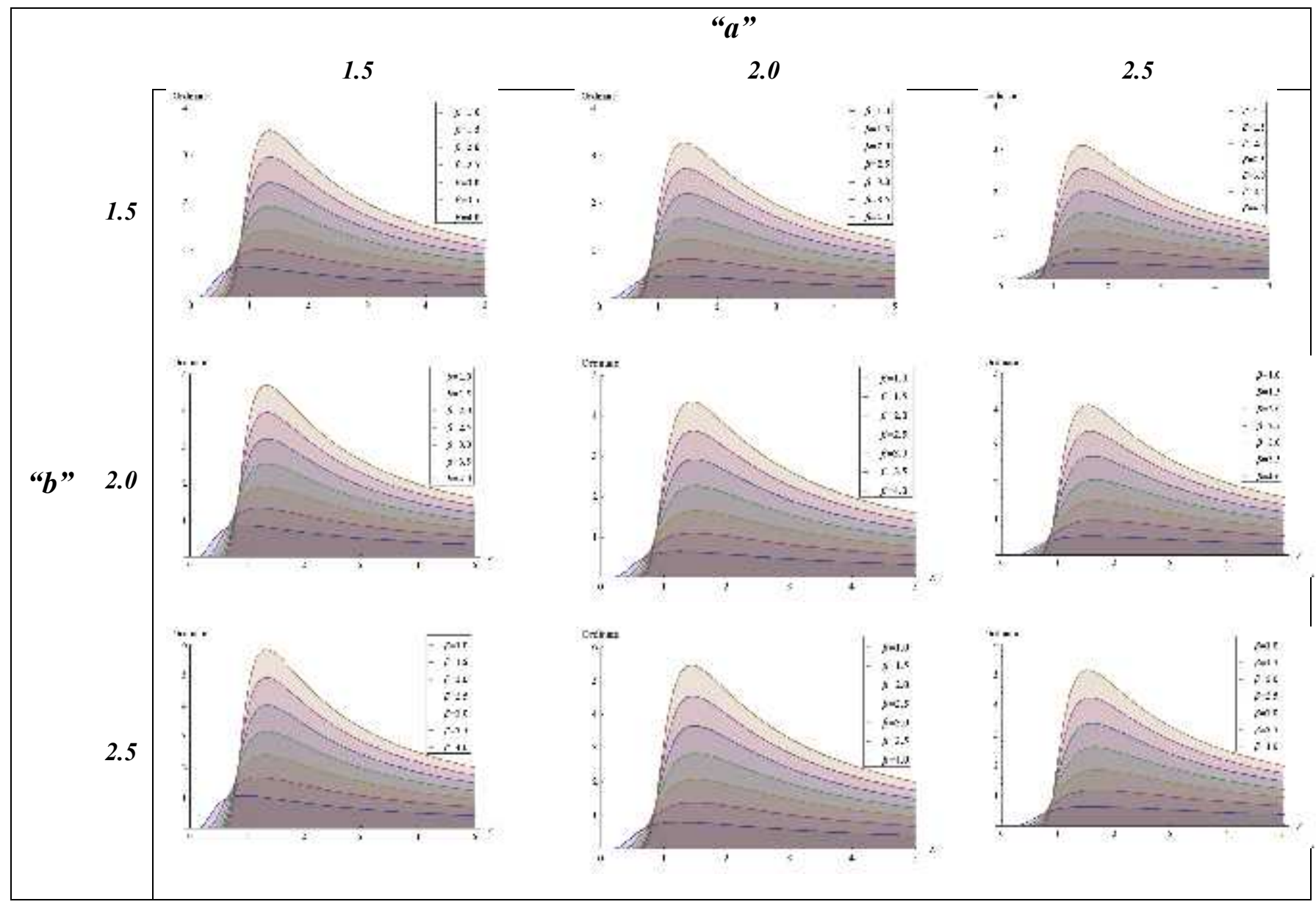

\section{Parameter Estimation}

In this section we have given the maximum likelihood estimates of parameter of KumIW distribution. The likelihood function for a random sample of size $n$ from density (2.3) is:

$$
L F=L\left(x_{1}, \ldots, x_{n}\right)=(a b \alpha \beta)^{n} \prod_{i=1}^{n} x_{i}^{-(\beta+1)} \exp \left(-a \alpha \sum_{i=1}^{n} x_{i}^{-\beta}\right) \prod_{i=1}^{n}\left[1-\exp \left(-a \alpha x_{i}^{-\beta}\right)\right]^{b-1}
$$


The Log of likelihood function is:

$$
\begin{aligned}
\ln (L F)=n \ln (a)+n \ln (b)+n \ln (\alpha)+n \ln (\beta)-(\beta+1) \sum_{i=1}^{n} \ln \left(x_{i}\right)-a \alpha \sum_{i=1}^{n} x_{i}^{-\beta} \\
+(b-1) \sum_{i=1}^{n} \ln \left[1-\exp \left(-a \alpha x_{i}^{-\beta}\right)\right]
\end{aligned}
$$

The derivatives of log of likelihood function w.r.t. the unknown parameters are:

$$
\begin{aligned}
& \frac{\partial \ln (L F)}{\partial a}=\frac{n}{a}-\alpha \sum_{i=1}^{n} x_{i}^{-\beta}+(b-1) \sum_{i=1}^{n} \frac{\alpha x_{i}^{-\beta} \exp \left(-a \alpha x_{i}^{-\beta}\right)}{1-\exp \left(-a \alpha x_{i}^{-\beta}\right)} \\
& \frac{\partial \ln (L F)}{\partial b}=\frac{n}{b} \sum_{i=1}^{n} \ln \left[1-\exp \left(-a \alpha x_{i}^{-\beta}\right)\right] \\
& \frac{\partial \ln (L F)}{\partial \alpha}=\frac{n}{\alpha}-a \sum_{i=1}^{n} x_{i}^{-\beta}+(b-1) \sum_{i=1}^{n} \frac{a x_{i}^{-\beta} \exp \left(-a \alpha x_{i}^{-\beta}\right)}{1-\exp \left(-a \alpha x_{i}^{-\beta}\right)} \\
& \frac{\partial \ln (L F)}{\partial \beta}=\frac{n}{\beta}-\sum_{i=1}^{n} \ln x_{i}+a \alpha \sum_{i=1}^{n}\left(\ln x_{i}\right) x_{i}^{-\beta}+(b-1) \sum_{i=1}^{n} \frac{a \alpha x_{i}^{-\beta}\left(\ln x_{i}\right) \exp \left(-a \alpha x_{i}^{-\beta}\right)}{1-\exp \left(-a \alpha x_{i}^{-\beta}\right)}
\end{aligned}
$$

The maximum likelihood estimates of $a, b, \alpha$ and $\beta$ can be obtained by solving equations (4.2) to (4.5) simultaneously. The observed information matrix for the parameters of Kum-IW distribution is given as:

$$
J(\boldsymbol{\theta})=-\left[\begin{array}{cccc}
J_{a a} & J_{a b} & J_{a \alpha} & J_{a \beta} \\
& J_{b b} & J_{b \alpha} & J_{b \beta} \\
& & J_{\alpha \alpha} & J_{\alpha \beta} \\
& & & J_{\beta \beta}
\end{array}\right] ;
$$

where $J_{\theta_{j} \theta_{k}}=\partial^{2} \ln (L F) / \partial \theta_{j} \partial \theta_{k}$. These entries are given in the Appendix.

\section{Application}

In this section we have given the real data application of Kum-IW distribution. We have fitted the Kum - IW distribution for two different data sets. The results are given in Table 5.1 below. The results of this table clearly indicates that the proposed Kum-IW distribution fits well the given data sets in comparison with other distributions as this distribution has smallest AIC values. 
Table 5.1: Parameter Estimates for Various Distributions

\begin{tabular}{|c|c|c|c|c|c|c|c|}
\hline & \multirow{2}{*}{ Model } & \multicolumn{4}{|c|}{ Parameters (SE in Parenthesis) } & \multirow{2}{*}{$\begin{array}{c}-2 \log \\
(\mathrm{LF})\end{array}$} & \multirow{2}{*}{ AIC } \\
\hline & & $\mathbf{a}$ & b & Alpha & Beta & & \\
\hline \multirow{5}{*}{ 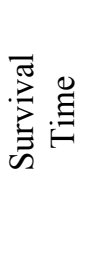 } & K-IW & $19.95(2.95)$ & $3195.14(92.14)$ & $0.45(0.07)$ & $0.73(0.02)$ & -7614.66 & -7606.66 \\
\hline & Inverse Weibull & & & $11.81(0.84)$ & $0.79(0.02)$ & 11848.51 & 11852.51 \\
\hline & Weibull & & & $52.38(0.99)$ & $1.61(0.04)$ & 11362.08 & 11366.08 \\
\hline & Gamma & & & $2.10(0.08)$ & $0.04(0.002)$ & 11392.86 & 11396.86 \\
\hline & K-IW & $837.09(95.95)$ & $6209.63(285.41)$ & $881.68(100.72)$ & $1.40(0.02)$ & 1807.73 & 1815.73 \\
\hline \multirow{3}{*}{$\frac{\vec{E}}{\sqrt[E]{n}}$} & Inverse Weibull & & & $485.72(83.30)$ & $0.68(0.02)$ & 10343.47 & 10347.47 \\
\hline & Weibull & & & $584.47(59.32)$ & $0.24(0.01)$ & 11869.48 & 11873.48 \\
\hline & Gamma & & & $2.10(0.08)$ & $0.04(0.002)$ & 11392.86 & 11396.86 \\
\hline
\end{tabular}

\section{References}

1. Cordeiro, G. M. and Castro, M. de (2010).A new family of generalized distributions. J. of Stat. Comp. and Sim . (2010). DOI: 10.1080/0094965YY.

2. Eugene, N., Lee, C. and Famoye, F. (2002). Beta-Normal distribution and its applications, Comm. in Statist: Theory and Methods, Vol. 31, 497-512.

3. Famoye, F., Lee, C. and Olumolade, O. (2005).The Beta-Weibull Distribution, Journal of Statistical Theory and Applications 4(2), 121-136.

4. Hanook, S., Shahbaz, M. Q., Mohsin, M. and Kibria, G. (2012). A Note on Beta Inverse Weibull Distribution, Comm. In Statist: Theory and Methods, To Appear.

5. Kumaraswamy, P. (1980).A generalized probability density function for doublebounded random processes, J. of Hydrology, Vol. 46, 79-88.

6. Nadarajah, S., Kotz, S. (2004). The Beta Gumbel Distribution, Mathematical Problems in Engineering,4, 323-332.

7. Nadarajah, S., Kotz, S. (2005).The Beta-Exponential Distribution, Reliability Engineering and System Safety 91, 689-697.

8. Prudnikov, A. P., Brychkov, Y. A. and Marichev, O. I. (1986).Integrals and Series. Vol. 3 Gordon and Breach Science Publishers, New York. 


\section{Appendix: Entries of Observed Information Matrix for Kum-IW Distribution}

$$
\begin{aligned}
& J_{a a}=\frac{\partial^{2} \ln (L F)}{\partial^{2} a}=-\frac{n}{a^{2}}-(b-1) \sum_{i=1}^{n} \frac{\alpha^{2} x_{i}^{-2 \beta} \exp \left(-a \alpha x_{i}^{-\beta}\right)}{\left\{1-\exp \left(-a \alpha x_{i}^{-\beta}\right)\right\}^{2}} \\
& J_{a b}=\frac{\partial^{2} \ln (L F)}{\partial a \partial b}=\sum_{i=1}^{n} \frac{\alpha x_{i}^{-\beta} \exp \left(-a \alpha x_{i}^{-\beta}\right)}{1-\exp \left(-a \alpha x_{i}^{-\beta}\right)} \\
& J_{a \alpha}=\frac{\partial^{2} \ln (L F)}{\partial a \partial \alpha}=-\sum_{i=1}^{n} x_{i}^{-\beta}-(b-1) \sum_{i=1}^{n} \frac{a \alpha x_{i}^{-2 \beta} \exp \left(-a \alpha x_{i}^{-\beta}\right)}{\left\{1-\exp \left(-a \alpha x_{i}^{-\beta}\right)\right\}^{2}}+(b-1) \sum_{i=1}^{n} \frac{x_{i}^{-\beta} \exp \left(-a \alpha x_{i}^{-\beta}\right)}{1-\exp \left(-a \alpha x_{i}^{-\beta}\right)} \\
& J_{a \beta}=\frac{\partial^{2} \ln (L F)}{\partial a \partial \beta}=\alpha \sum_{i=1}^{n}\left(\ln x_{i}\right) x_{i}^{-\beta}+(b-1) \sum_{i=1}^{n} \frac{a \alpha^{2}\left(\ln x_{i}\right) x_{i}^{-2 \beta} \exp \left(-a \alpha x_{i}^{-\beta}\right)}{\left\{1-\exp \left(-a \alpha x_{i}^{-\beta}\right)\right\}^{2}} \\
& -(b-1) \sum_{i=1}^{n} \frac{\alpha\left(\ln x_{i}\right) x_{i}^{-\beta} \exp \left(-a \alpha x_{i}^{-\beta}\right)}{1-\exp \left(-a \alpha x_{i}^{-\beta}\right)} \\
& J_{b b}=\frac{\partial^{2} \ln (L F)}{\partial^{2} b}=-\frac{n}{b^{2}} ; \quad J_{b \alpha}=\frac{\partial^{2} \ln (L F)}{\partial b \partial \alpha}=\sum_{i=1}^{n} \frac{a x_{i}^{-\beta} \exp \left(-a \alpha x_{i}^{-\beta}\right)}{1-\exp \left(-a \alpha x_{i}^{-\beta}\right)} \\
& J_{b \beta}=\frac{\partial^{2} \ln (L F)}{\partial b \partial \beta}=-\sum_{i=1}^{n} \frac{a \alpha\left(\ln x_{i}\right) x_{i}^{-\beta} \exp \left(-a \alpha x_{i}^{-\beta}\right)}{1-\exp \left(-a \alpha x_{i}^{-\beta}\right)} \\
& J_{\alpha \alpha}=\frac{\partial^{2} \ln (L F)}{\partial^{2} \alpha}=-\frac{n}{\alpha^{2}}-(b-1) \sum_{i=1}^{n} \frac{a^{2} x_{i}^{-2 \beta} \exp \left(-a \alpha x_{i}^{-\beta}\right)}{\left\{1-\exp \left(-a \alpha x_{i}^{-\beta}\right)\right\}^{2}} \\
& J_{\alpha \beta}=\frac{\partial^{2} \ln (L F)}{\partial \alpha \partial \beta}=a \sum_{i=1}^{n}\left(\ln x_{i}\right) x_{i}^{-\beta}+(b-1) \sum_{i=1}^{n} \frac{a^{2} \alpha\left(\ln x_{i}\right) x_{i}^{-2 \beta} \exp \left(-a \alpha x_{i}^{-\beta}\right)}{\left\{1-\exp \left(-a \alpha x_{i}^{-\beta}\right)\right\}^{2}} \\
& +(b-1) \sum_{i=1}^{n} \frac{a\left(\ln x_{i}\right) x_{i}^{-\beta} \exp \left(-a \alpha x_{i}^{-\beta}\right)}{1-\exp \left(-a \alpha x_{i}^{-\beta}\right)} \\
& J_{\beta \beta}=\frac{\partial^{2} \ln (L F)}{\partial^{2} \beta}=-\frac{n}{\beta^{2}}-a \alpha \sum_{i=1}^{n}\left(\ln x_{i}\right)^{2} x_{i}^{-\beta}-(b-1) \sum_{i=1}^{n} \frac{a^{2} \alpha^{2}\left(\ln x_{i}\right)^{2} x_{i}^{-2 \beta} \exp \left(-a \alpha x_{i}^{-\beta}\right)}{\left\{1-\exp \left(-a \alpha x_{i}^{-\beta}\right)\right\}^{2}} \\
& +(b-1) \sum_{i=1}^{n} \frac{a \alpha\left(\ln x_{i}\right)^{2} x_{i}^{-\beta} \exp \left(-a \alpha x_{i}^{-\beta}\right)}{1-\exp \left(-a \alpha x_{i}^{-\beta}\right)}
\end{aligned}
$$

